\title{
052 6CIT FOR DETECTION OF DEMENTIA AND COGNITIVE IMPAIRMENT
}

Khaled Abdel-Aziz, Andrew Larner. Walton Centre Foundation Trust

\subsection{6/jnnp-2014-309236.52}

Objective To report the diagnostic utility of the Six-Item Cognitive Impairment Test (6CIT), a brief (2-3 minute) cognitive screening instrument, for the detection of dementia and mild cognitive impairment.

Method/Setting Prospective observational study, Cognitive Function Clinic.

Results Of 100 patients tested (M:F=52:48; age range 23-94 years, median 58.5 years) over a 5-month period (July-November 2013), 21 had dementia by DSM-IV-TR criteria and 20 had MCI. 6CIT and MMSE scores in a subgroup of patients $(n=61)$ showed a moderate negative correlation $(r=$ -0.69). 6CIT was found to have good sensitivity and specificity for the diagnosis of dementia ( 0.80 and 0.79 respectively). Sensitivity for diagnosis of any cognitive impairment (dementia and MCI) was good (0.80) but specificity less so (0.69). Area under the ROC curve, a measure of diagnostic accuracy, was 0.88 and 0.83 for diagnosis of dementia and of any cognitive impairment respectively.

Conclusions 6CIT had good sensitivity and specificity for diagnosis of dementia. However, extending its scope to MCI diagnosis showed poorer specificity although sensitivity remained high. 6CIT might prove useful for patient assessment in dedicated cognitive disorders clinics. 